\title{
Perfis clínico e laboratorial de cães de abrigo submetidos a esterilização*
}

\section{Evaluation of clinical and laboratorial profiles of shelter dogs submitted to sterilizations}

\author{
Carine Budziak, ${ }^{* *}$ Amanda Anater, ${ }^{* * *}$ José Ademar Villanova Junior, ${ }^{* * *}$ Rita Maria Venancio Mangrich, ${ }^{* * * *}$ \\ Dariane Cristina Catapan, ${ }^{* * * * *}$ Cláudia Turra Pimpão*******
}

\begin{abstract}
Resumo
Avaliou-se o perfil clínico e laboratorial de 90 cães de abrigos submetidos à ovariohisterectomia e orquiectomia. A avaliação préoperatória foi realizada por exame clínico, hemograma, coagulograma, coproparasitológico, pesquisa de hemoparasitas, urinálise e exames bioquímicos. Dos animais participantes, 53,33\% eram fêmeas; $93,3 \%$ eram sem raça definida (SRD); a idade média foi de 3,56 $\pm 1,32$ anos e peso médio de 10,87 $\pm 6,08 \mathrm{~kg} ; 65,55 \%$ apresentaram escore corporal normal e 42,22\% foram considerados cães dóceis. No exame clínico geral, as principais alterações foram da temperatura retal e frequência respiratória, no exame específico foi observado pulicilose, prurido, seborréia e dermatites. Verificou-se anemia em $18,60 \%$ dos animais e sua intensidade apresentou correlação com o escore corporal, mas esta correlação não foi observada com a presença de hipoalbuminemia. Os valores de contagem de leucócitos, neutrófilos e bastonetes estavam elevados, porém decorrentes de estresse. Na urinálise, as principais alterações encontradas foram na densidade, aspecto e presença de bactérias. Ao exame coproparasitológico, $65,45 \%$ dos animais foram diagnosticados com Ancylostoma sp. e Trichuris sp. Os cães de abrigo apresentaram alterações nos exames realizados, sugerindo que possuem perfis clínico e laboratorial próprios, pois nenhuma alteração foi sugestiva de patologia, provavelmente por uma condição de estresse e deficiência nutricional.
\end{abstract}

Palavras-chave: animais domésticos, contagem de células sanguíneas, castração, urinálise.

\begin{abstract}
We evaluated the clinical and laboratory profile of 90 dogs shelters undergoing ovariohysterectomy and orchiectomy. Preoperative evaluation was performed by clinical examination, blood tests, coagulation tests, fecal, hemoparasites research, urinalysis and biochemical tests. Participants animals, $53.33 \%$ were female; $93.3 \%$ were mixed breed (SRD); the mean age was $3.56 \pm 1.32$ years and average weight of $10.87 \pm 6.08 \mathrm{~kg} ; 65.55 \%$ had normal body score and $42.22 \%$ were considered friendly dogs. In general clinical examination, the main changes were the rectal temperature and respiratory rate, the specific examination was observed pulicilose, itching, seborrhea and dermatitis. It was $18.60 \%$ anemia in animal and its intensity was correlated with the body condition score, but this correlation was not observed with the presence of hypoalbuminemia. The leukocyte count values Neutrophils and rods were high, but due to stress. In urinalysis, the main changes were found in the density, appearance and presence of bacteria. When fecal examination, $65.45 \%$ of the animals were diagnosed with Ancylostoma sp. and Trichuris sp. Shelter dogs showed changes in the blood tests, suggesting that have clinical and laboratory profiles themselves, because no changes were suggestive of pathology, probably a stress condition and nutritional deficiency.
\end{abstract}

Keywords: domestic animal, blood cell count, castration, urinalysis.

\section{Introdução}

As instituições de abrigo são organizações privadas, nãogovernamentais que podem desenvolver atividades com o apoio do governo, sem visar a obtenção de lucro. Um abrigo para animais é um local que aloja diversos cães que em geral são animais errantes, capturados ou abandonados. Dos muitos problemas encontrados nesses abrigos, pode-se citar a superpopulação, falta de recursos, alimentação não balanceada, gerando deficiências nutricionais, falta de atendimento veterinário, o que resulta em doenças infecciosas e parasitárias (Cerqueira, 2012).

Dessa maneira, ambientes de abrigo devem ser enriquecidos para prevenir o estresse severo e incidência de doenças, assim como esses animais devem ser esterilizados com objetivo de

${ }^{*}$ Recebido em 24 de junho de 2016 e aceito em 26 de outubro de 2016

**Prefeitura Municipal de Araucária. M.V., Mestre em Ciência Animal pela Pontifícia Universidade Católica do Paraná (PUCPR) - Curitiba, PR, Brasil.

***Pontifícia Universidade Católica do Paraná. M.V., doutoranda em Saúde, Tecnologia e Produção Animal Integrada pela PUCPR.

****Pontifícia Universidade Católica do Paraná. M.V., MSc, PhD, Professor Adjunto de Cirurgia na PUCPR.

*****Pontifícia Universidade Católica do Paraná. M.V., MSc, Professora Adjunta de Patologia Clínica na PUCPR.

******Pontifícia Universidade Católica do Paraná. M.V., MSc, Doutoranda em Saúde, Tecnologia e Produção Animal Integrada pela PUCPR.

*******Pontifícia Universidade Católica do Paraná. M.V., MSc, PhD, Professora Titular de Farmacologia e Toxicologia Veterinária na PUCPR. Autor para correspondência: claudia.pimpao@pucpr.br 
controlar a população e serem doados mais rapidamente(Miller, 2004).

Pacientes cirúrgicos, mesmo aqueles que são submetidos a procedimentos de ovario-histerectomia e orquiectomia (eletivos e de baixo risco), requerem atenção e devem passar por um exame físico completo, seguido de exame laboratorial apropriado. Também deve-se observar a condição geral do animal, incluindo escore corporal, temperamento, entre outros(FOSSUM, 2005).

A esterilização de cães de abrigo é um desafio ao médicoveterinário, uma vez que esses animais podem apresentar perfis clínico e laboratorial próprios e, na maioria das vezes, não são realizados exames pré-operatórios, pois, geralmente as limitações financeiras são muitas. Assim, o objetivo do presente trabalho foi avaliar o perfil clínico e laboratorial de cães de abrigo submetidos à ovário-histerectomia e orquiectomia.

\section{Material e métodos}

Foi realizada na Unidade Hospitalar de Animais de Companhia (UHAC) da Pontifícia Universidade Católica do Paraná (PUCPR) uma campanha de castração, na qual 90 animais escolhidos ao acaso provenientes da Organização não governamental denominada de Associação São Francisco de Assis de Proteção Animal (ASFAPA), localizada no Município de Balsa Nova, na região metropolitana de Curitiba, participaram. Este trabalho foi aprovado pelo Comitê de Ética no Uso de Animais (CEUA) da PUCPR (protocolo $n^{\circ}$ 409/2009).

Os animais foram avaliados pelo exame clínico completo, sendo coletadas amostras de sangue, urina e fezes para avaliação laboratorial.

Após a identificação do paciente (data, nome, espécie, idade, raça e sexo) dava-se início ao exame clínico. Devido ao fato desses pacientes serem provenientes de abrigo, a anamnese não era preenchida, já que não possuíam proprietários e nem histórico.

O exame clínico era realizado por meio da avaliação dos seguintes parâmetros: peso, escore corporal (1= caquético, $2=$ magro, $3=$ normal, $4=$ sobrepeso e $5=$ obeso) (Nobre et al., 2010), temperamento do paciente (1= dóceis, $2=$ agressivos, $3=$ agitados e 4= assustados), temperatura retal, frequência cardíaca e respiratória (por método auscultatório), tempo de preenchimento capilar (TPC), condições de hidratação e coloração de mucosas. Também foi realizada a avaliação clínica específica por sistemas: tegumentar, oftálmico e complexo auditivo; respiratório, cardiocirculatório, digestório, geniturinário, osteomuscular e neurológico.

Considerou-se cão caquético, aquele que dotava de proeminências ósseas visíveis, ausência de cobertura de gordura e contratura abdominal muito evidente; cão magro o que tinha costelas facilmente palpáveis, mínima cobertura de gordura e contratura abdominal evidente; cão com escore corporal normal aquele com costelas palpáveis, mas não visíveis, presença de cintura e abdômen contraído; cão com sobrepeso aquele com costelas palpáveis, porém com leve excesso de cobertura de gordura e cintura de difícil visualização e cão obeso o qual tinha costelas de difícil palpação, com espessa camada de gordura, depósito de gordura em área lombar e base da cauda, ausência de cintura e abdômen distendido (Nobre et al., 2010).
Classificou-se como dóceis, animais que permitiam o manejo e havia ausência de agressividade; agressivos aqueles que não permitiam o manejo e demonstravam sinais como: rosnados, latidos furiosos e tentativa de mordedura; agitados aqueles que não estavam acostumados com o manejo, eram arredios e poderiam demonstrar sinais de agressividade e os assustados eram os hiperativos, havia dificuldade para o manejo, porém não eram agressivos. No entanto, os assustados eram animais que necessitavam de uma abordagem diferente, a qual deveria ser realizada com cuidado e demonstrações de carinho e confiança ao animal. Dessa maneira, o animal permitia o manejo e a realização das atividades do experimento.

Foram excluídas do experimento todas as fêmeas prenhes e as que apresentavam sinais de estro. $O$ critério para que o animal fosse submetido ao procedimento cirúrgico foi o exame clínico, pois simularia uma condição de ambiente de campanha de castração, onde em muitos casos a avaliação laboratorial não é realizada devido aos escassos recursos financeiros.

Após a realização do exame clínico, eram iniciadas as coletas de amostras de sangue, urina e fezes, as quais eram submetidas aos exames laboratoriais no Laboratório de Patologia Clínica da UHAC da PUCPR. As coletas de amostras de sangue eram realizadas por meio da punção venosa jugular e a coleta de urina por meio de cistocentese.

Os exames laboratoriais pré-operatórios realizados duran-te o experimento foram: hemograma, coagulograma, coproparasitológico, pesquisa de hemoparasitas, urinálise e bioquímicos que constituíram da dosagem de alanina aminotransferase $(A L T)$, fosfatase alcalina (FA), ureia, creatinina, albumina, fibrinogênio, transaminase oxalacética (TGO), gama glutamil transferase (GGT), proteínas totais, cálcio, fósforo, cálcio iônico, potássio iônico, sódio iônico e glicemia. Os resultados do hemograma e exames bioquímicos foram baseados no padrão de normalidade segundo Kaneko et al. (1997). Os valores de tempo de protrombina (TP), tempo de tromboplastina parcial ativada (TTPA) e dosagem de fibrinogênio foram mensurados.

O protocolo anestésico selecionado para o procedimento foi o uso de anestesia injetável dissociativa. Outros fármacos, como anti-inflamatórios não esteroides, benzodiazepínicos e anestésicos locais, foram adicionados a este protocolo objetivando a utilização dos princípios de analgesia preemptiva e analgesia multimodal, caracterizando uma anestesia balanceada. Como medicação pré anestésica foi utilizado de forma intramuscular atropina $(0,022 \mathrm{mg} / \mathrm{kg})$ e cetoprofeno (1 $\mathrm{mg} / \mathrm{kg})$, após 15 minutos, quetamina $(15 \mathrm{mg} / \mathrm{kg})$ e xilazina $(0,2$ $\mathrm{mg} / \mathrm{kg}$ ). Na indução e manutenção anestésica, foi utilizado de forma intravenosa quetamina $(7 \mathrm{mg} / \mathrm{kg})$ e midazolam $(0,5 \mathrm{mg} /$ $\mathrm{kg})$. A anestesia local foi realizada utilizando-se lidocaína $2 \%$ sem vasoconstritor. Nas fêmeas foi utilizado $1 \mathrm{ml} / 5 \mathrm{~kg}$, por via epidural (volume máximo de $5 \mathrm{ml}$ ). Nos machos a dose usada foi 0,5 a $1 \mathrm{ml}$, de forma intratesticular.

Durante todo o procedimento os animais foram mantidos em fluidoterapia, entubados e conectados a fonte de oxigênio a $100 \%$. Os procedimentos cirúrgicos foram realizados com rigorosa técnica asséptica de acordo com o que é preconizado por Fossum (2005). A monitoração anestésica ocorreu a partir do momento da aplicação da medicação pré-anestésica (MPA) até a completa recuperação do paciente no pós-operatório. 
Foi administrado antibiótico (Enrofloxacina $5 \mathrm{mg} / \mathrm{kg}$ ), por via intramuscular, durante o trans-operatório em todos os animais participantes do experimento. A ocorrência de hemorragia durante a cirurgia foi registrada.

Os pacientes ficaram internados para acompanhamento do período pós-operatório por seis dias após o procedimento. Neste período, foi administrado Enrofloxacina $5 \mathrm{mg} / \mathrm{kg}$ a cada 12 horas por 7 dias e Meloxicam $0,1 \mathrm{mg} / \mathrm{kg}$ a cada 24 horas por 4 dias, foram alimentados com ração comercial e água ad libitum. Após esse período os animais foram devolvidos ao abrigo.

Para a apresentação dos resultados foi utilizado estatística descritiva, onde os dados estão expressos na forma de média, desvio padrão e porcentagem. Para a análise estatística de alguns parâmetros (índice de escore corporal com intensidade de anemia, de hipoalbuminemia, relação de animais anêmicos e presença de parasitose, e, relação de animais trobocitopênicos e hemorragias), foi utilizado o teste de correlação (Spearman). O nível de significância adotado foi $5 \%(p=0,05)$. Todos os cálculos foram realizados utilizando o Software estatístico GraphPadPrismversion 5.00 for Mac., San Diego - Califórnia, EUA.

\section{Resultados e discussão}

Verificou-se que, dos 90 animais participantes, $53,33 \%$ eram fêmeas, e $46,66 \%$ eram machos. Em relação às raças dos animais, 93,3\% eram sem raça definida (SRD), seguidos de Pit Bull $(2,22 \%)$, Boxer $(1,11 \%)$, Labrador $(1,11 \%)$, Pinscher $(1,11 \%)$ e Poodle $(1,11 \%)$. A idade média dos animais foi de $3,56 \pm 1,32$ anos e o peso médio de 10,87 $\pm 6,08 \mathrm{~kg}$. Em relação ao escore corporal, conforme Figura 1, a maioria dos animais apresentou escore corporal normal $(65,55 \%)$, seguido de cães magros $(30 \%)$, cães com sobrepeso $(3,33 \%)$ e apenas um cão caquético $(1,11 \%)$. Não foram observados cães obesos. Em relação ao temperamento, $42,22 \%$ foram considerados cães dóceis, $4,44 \%$ agressivos, $18,88 \%$ agitados e $34,44 \%$ assustados (Figura 2).

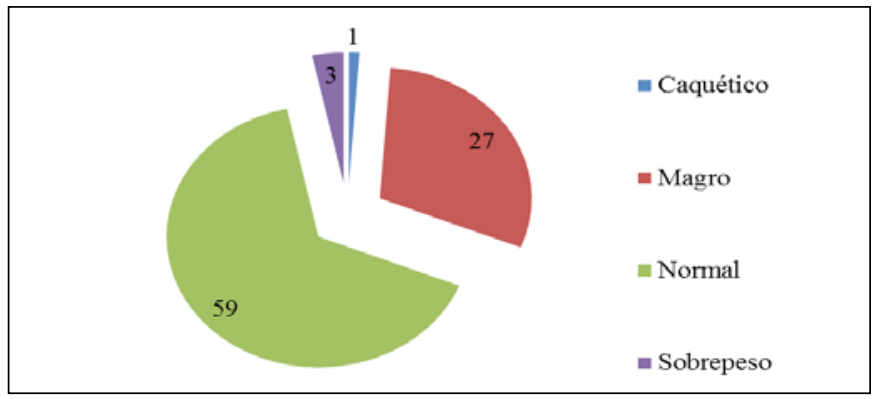

Figura 1: Condição corporal dos cães de abrigo submetidos à campanha de castração, na UHAC em 2009, baseada no escore corporal variando na escala de 1 (um) a 5 (cinco), conforme Nobre et al. (2010) $(n=90)$

Pelo exame clínico geral, verificou-se aumento da temperatura retal $(38,6 \pm 0,6)$ em $17,77 \%$ dos cães avaliados e também

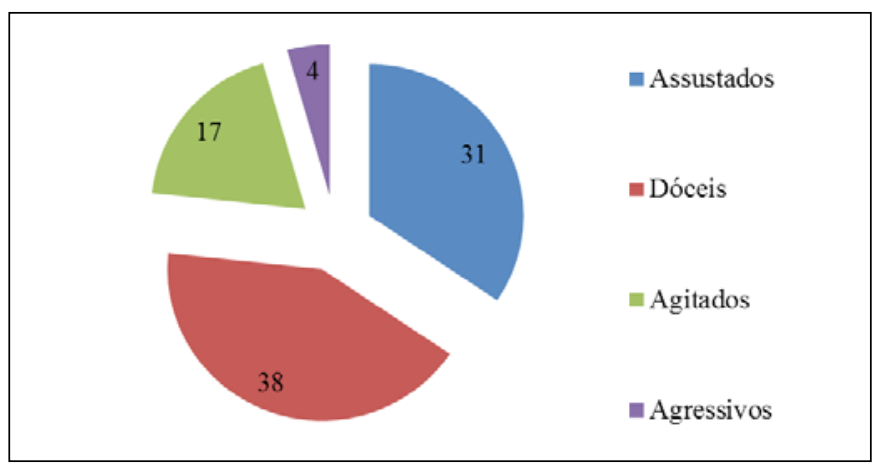

Figura 2: Temperamento dos cães de abrigo submetidos à campanha de castração, na UHAC em 2009 ( $n=90)$

da frequência respiratória $(33,72 \pm 16,23)$ em $34,44 \%$. Ambas alterações foram registradas em animais que apresentavam temperamento agitado ou assustado. A ansiedade pode estar relacionada ao aumento da temperatura retal e também pode provocar o aumento da frequência respiratória e cardíaca (Yamamoto et al., 2012).

O TPC avaliado nos cães apresentou 4,44\% de alteração em relação aos valores normais. Além disso, $15,55 \%$ dos cães apresentavam-se desidratados e $7,77 \%$ possuíam alteração na coloração das mucosas, sendo que $6,66 \%$ eram rósea clara e $1,11 \%$ rósea escura.

As principais alterações relatadas ao exame clínico específico foram relativas ao sistema tegumentar. Dentre as alterações estavam: puliciose, prurido, seborréia, pelagem opaca e quebradiça e dermatites. Alguns cães apresentavam otites e gengivites. Aavaliação do sistema cardiorrespiratório demonstrou que alguns animais apresentavam sons broncovesiculares e arritmia sinusal. $\mathrm{Na}$ avaliação específica dos outros sistemas não foram encontradas alterações dignas de nota.

As análises laboratoriais foram realizadas em todos os cães participantes do experimento. Porém, para alguns exames (n) as amostras estavam inviáveis, impossibilitando a realização do mesmo.

A Tabela 1 apresenta os valores de hemograma e a frequência de alterações em relação aos parâmetros de normalidade. Podese observar alterações na contagem de eritrócitos, hemoglobina, hematócrito, volume globular médio (VGM) e concentração de hemoglobina globular média (CHGM). As alterações no número de eritrócitos, concentração de hemoglobina e/ou hematócrito abaixo dos valores de referência são caracterizados como anemia (Lopes et al., 2007). E os valores de VGM e CHGM abaixo dos padrões de normalidade caracterizam anemia microcítica e hipocrômica (Drumond, 2013) dos animais, o que provavelmente está relacionada à deficiência nutricional $\mathrm{e}$ endoparasitoses. O teste de correlação entre animais anêmicos e escore corporal apresentou significância $(p<0,05 ; n=16)$. Portanto, o índice de escore corporal, pode estar relacionado com a intensidade da anemia. 
Tabela 1: Média e desvio padrão dos valores de hemograma e frequência de alterações em relação aos parâmetros de normalidade em cães submetidos às campanhas de castração, na UHAC em 2009 (n=86)

\begin{tabular}{lccc}
\hline Hemograma & $\mathrm{X} \pm \mathrm{S}$ & $f(\%)$ & Parâmetros Normais $^{1}$ \\
\hline Eritrócitos & $6.791 \pm 990$ & $18,60 \downarrow$ & $5.500-8.50010^{6} / \mu \mathrm{L}$ \\
Hemoglobina & $13,58 \pm 3,76$ & $32,56 \downarrow$ & $12-18 \mathrm{~g} / \mathrm{dL}$ \\
Hematócrito & $42 \pm 6$ & $18,60 \downarrow$ & $37-55 \%$ \\
VGM & $63,05 \pm 9,03$ & $51,16 \downarrow$ & $60-77 \mathrm{fL}$ \\
CHGM & $32,75 \pm 11,16$ & $79,06 \downarrow$ & $32-36 \%$ \\
Plaquetas & $256.618 \pm 102.723$ & $44,19 \downarrow$ & $200.000-500.00010^{5} / \mu \mathrm{L}$ \\
Leucócitos & $13.683 \pm 4.492$ & $23,25 \uparrow$ & $6000-17000$ quant./ $\mu \mathrm{L}$ \\
Eosinófilos & $1.408 \pm 1.050$ & $58,14 \uparrow$ & $100-1250$ quant./ $\mu \mathrm{L}$ \\
Linfócitos & $2.609 \pm 1.103$ & 10,46 & $1000-4800$ quant. $/ \mu \mathrm{L}$ \\
Monócitos & $1.059 \pm 567$ & $32,56 \uparrow$ & $150-1350$ quant./ $\mu \mathrm{L}$ \\
Bastonetes & $617 \pm 817$ & $58,14 \uparrow$ & $0-300$ quant./ $\mu \mathrm{L}$ \\
Segmentados & $8.029 \pm 3.389$ & $17,44 \uparrow$ & $3000-11500$ quant./ $\mu \mathrm{L}$ \\
Neutrófilos & $8.646 \pm 3.817$ & $22,09 \uparrow$ & $3000-11800$ quant./ $\mu \mathrm{L}$ \\
Proteína Plasmática & $6,9 \pm 0,7$ & $18,60 \downarrow$ & $6-8$ g/dL \\
\hline
\end{tabular}

$\downarrow$ : valor abaixo da normalidade

$\uparrow:$ valor acima da normalidade

'Kaneko et al. (1997)

A contagem de plaquetas demonstrou valores abaixo da referência (trombocitopenia) A diminuição do número de plaquetas pode levar a alterações de hemostasia em cães (Fossum, 2005). Porém, o teste de correlação entre os animais trombocitopênicos e hemorragias durante o período transoperatório não apresentou significância ( $p>0,05 ; n=25)$. Possivelmente, a trombocitopenia não seja a única causa de hemorragias durante as cirurgias, uma vez que $12,22 \%$ dos cães apresentaram hemorragias durante o período transoperatório.

A contagem do número de leucócitos, neutrófilos e bastonetes, apresentou aumento em relação aos valores de referência. A leucocitose pode ter causas fisiológicas, reativas ou proliferativas (Lopes et al., 2007). O número de segmentados era superior aos neutrófilos imaturos, indicando desvio à esquerda regenerativo (Drumond, 2013). Logo, essas alterações podem estar relacionadas às infecções crônicas ou moderadas descritas no exame clínico, caracterizando leucocitose reativa. Porém, os animais com leucocitose e neutrofilia não apresentavam febre. Portanto, os valores de normalidade de neutrófilos bastonetes e segmentados para cães de abrigo, podem ser maiores quando comparados aos da literatura (Kaneko et al., 1997; Drumond, 2013).

A eosinofilia pode ocorrer devido à perda tecidual crôni-ca, especialmente reações alérgicas; parasitismo, hipoadrenocorticismo; terapia medicamentosa; estro em cadelas; predisposição racial; desordens purulentas e eosinofilia reacional (Thrall, 2007). A eosinofilia observada neste trabalho pode ser explicada principalmente pelo intenso parasitismo, pois, por se tratar de animais de abrigo e sem histórico, não há informações suficientes para comprovar as outras causas descritas.

A monocitose observada nesses animais possivelmente está relacionada com as causas de leucocitose e neutrofilia, ou ainda, ao estresse severo que esses cães são submetidos no ambiente em que vivem (Lopes et al., 2007).

A hipoproteinemia pode estar relacionada, principalmente, com a dieta mal balanceada com níveis baixos de proteínas e a presença de parasitas intestinais (Lopes et al., 2007; Thrall, 2007).

No coagulograma, os valores de tempo de protrombina (TP) e tempo de tromboplastina parcial ativada (TTPA) apresentaram-se acima dos padrões de normalidade (TP: 5,47 -8,27"; TTPA: 13,5 - 16,7"), segundo Lopes et al. (2005), em $31,50 \%$ e $16,43 \%$ dos casos respectivamente $(n=73)$. A média e o desvio padrão de TP foram 8,0 e $\pm 2,1$ e de TTPA 11,8 e $\pm 4,5$, respectivamente. Nesse caso (não ocorreu aumento de TP e TTPA juntamente) sugere-se que as alterações não estão relacionadas a distúrbios da via comum da cascata de coagulação, mas sim deficiência de fatores de coagulação, coagulação intravascular disseminada (CIVD) ou veneno cumarínico. Porém, a avaliação para desordens hemostáticas depende de uma história clínica detalhada e de exame físico específico, que no caso dos animais de abrigo, essa avaliação ficou comprometida (Lopes et al., 2007).

No entanto, a deficiência nutricional apresentada pelos animais, principalmente relacionadas à hipocalcemia e hipoproteinemia, pode ser responsável pela deficiência de fatores de coagulação e como consequência pelos distúrbios hemorrágicos. Os valores de referência para TP e TTPA encontrados em literatura para o plasma canino sadio, utilizando reagentes comerciais, varia significativamente (Lopes et al., 2005). O TP tem uma variação de 5,8 a 14,9 segundos e o TTPA pode variar de 11 a 25 segundos (Lopes et al., 2005). Considerando essa variação de valores, os tempos encontrados como resultados podem não estar alterados.

Os valores das análises bioquímicas estão descritos nas Tabelas 2 e , conforme o tipo de exame realizado e o número de amostras viáveis.

Tabela 2: Média e desvio padrão das análises bioquímicas e frequência de alterações em relação aos parâmetros normais em cães submetidos às campanhas de castração, na UHAC em 2009 ( $n=90)$

\begin{tabular}{lccc}
\hline Bioquímicos & $\begin{array}{c}\% \text { de } \\
\text { alterações }\end{array}$ & $\mathrm{X} \pm \mathrm{S}$ & $\begin{array}{c}\text { Parâmetros Normais } \\
\text { (Kaneko et al., 1997) }\end{array}$ \\
\hline ALT & 4,44 & $37,76 \pm 23,93$ & $0-102 \mathrm{U} / \mathrm{L}$ \\
FA & 0 & $46,83 \pm 24,58$ & $0-156 \mathrm{U} / \mathrm{L}$ \\
Uréia & $36,66 \downarrow$ & $27,34 \pm 12,40$ & $21-60 \mathrm{mg} / \mathrm{dL}$ \\
Creatinina & 3,33 & $1,03 \pm 0,22$ & $0,5-1,5 \mathrm{mg} / \mathrm{dL}$ \\
Albumina & $56,66 \downarrow$ & $2,48 \pm 0,60$ & $2,6-3,3 \mathrm{~g} / \mathrm{L}$ \\
\hline
\end{tabular}

A presença de hipoalbuminemia pode ser decorrente de perda seletiva através de doença renal e gastrintestinal, decréscimo 
de síntese de albumina através de hepatopatia e má nutrição (Amaral et al., 1995; Lopes et al., 2007). O teste de correlação entre escore corporal e hipoalbuminemia não apresentou significância $(p>0,05 ; n=51)$. Apesar de $65,55 \%$ dos animais apresentarem escore corporal considerado normal, os valores dos exames laboratoriais indicam deficiência nutricional. Além disso, eram alimentados uma vez ao dia, com alimento composto por ração para cães de baixa qualidade nutricional ou farinha de carne e ossos, misturados a pão, farinha de milho, resíduo de óleo vegetal, restos de comida e água. A alimentação era composta basicamente por carboidratos e fibras.

Os valores de ureia apresentam-se alterados decorrente de doenças hepáticas, dietas com baixa quantidade de proteínas e hipoproteinemia (Thrall, 2007). Acredita-se que a baixo valor de ureia esteja relacionado à deficiência de proteína na dieta.

Conforme Tabela 3, a mensuração de proteínas totais descrita na análise bioquímica apresentou aumento comparado aos padrões de normalidade. $O$ método de análise colorimétrico pode sofrer alterações em sua interpretação, devido à qualidade do soro analisado. Se o soro apresenta-se hemolisado, ictérico ou lipêmico poderia ocorrer o aumento do valor, conforme descrito no Kit Labtest $₫$ (2014). Várias amostras desse trabalho apresentavam-se hemolisadas. Além disso, esperava-se que a dosagem de proteínas totais fosse baixa, pelo fato de constatar hipoalbuminemia e hipoproteinemia plasmática. Por esses motivos, acredita-se que os resultados da dosagem de proteínas totais foram mascarados.

Os baixos valores de cálcio, cálcio iônico, fósforo, sódio iônico e potássio iônico podem estar relacionados à má nutrição, pois esses minerais são adquiridos através da dieta (Beitz et al., 2006). Além disso, os valores de cálcio total podem sofrer interferência dos valores séricos de albumina, pois essa proteína é carreadora de cálcio (Lustoza et al., 2005).

Tabela 3: Porcentagem de alterações em relação aos parâmetros normais, média e desvio padrão dos valores de AST, GGT, proteína total, cálcio, cálcio iônico, fósforo, sódio iônico, potássio iônico e glicemia de cães submetidos às campanhas de castração, na UHAC em $2009(n=36)$

\begin{tabular}{lccc}
\hline Bioquímicos & $\%$ de alterações & $X \pm S$ & $\begin{array}{c}\text { Parâmetros Normais } \\
\text { (Kaneko et al., 1997) }\end{array}$ \\
\hline AST & 0 & $27 \pm 8$ & $0-66 \mathrm{U} / \mathrm{L}$ \\
GGT & 2,78 & $5,66 \pm 1,27$ & $0-10 \mathrm{U} / \mathrm{L}$ \\
Proteína Total & $52,78 \uparrow$ & $7,85 \pm 4,30$ & $5,4-7,1 \mathrm{~g} / \mathrm{L}$ \\
Cálcio & $100 \downarrow$ & $6,75 \pm 1,13$ & $9-11,3 \mathrm{mg} / \mathrm{dL}$ \\
Cálcio lônico & $13,88 \downarrow$ & $4,79 \pm 0,37$ & $5,2-6,0 \mathrm{mg} / \mathrm{dL}$ \\
Fósforo & $30,55 \downarrow$ & $3,86 \pm 1,81$ & $2,6-6,2 \mathrm{mg} / \mathrm{dL}$ \\
Sódio lônico & $38,88 \downarrow$ & $141 \pm 2,65$ & $141-152 \mathrm{mmol} / \mathrm{L}$ \\
Potássio lônico & $66,66 \downarrow$ & $4,2 \pm 0,5$ & $4,4-5,3 \mathrm{mmol} / \mathrm{L}$ \\
Glicemia & 5,55 & $86,5 \pm 24,96$ & $65-118 \mathrm{mg} / \mathrm{dL}$ \\
\hline
\end{tabular}

Em relação à dosagem de fibrinogênio, obteve-se $16,66 \%$ de alterações abaixo dos parâmetros normais $(n=30)$, a média e desvio padrão foram iguais a $210 \pm 84,49$, considerando como parâmetro normal a dosagem de $200-500$ mg/dL (Kaneko et al., 1997). Essa alteração pode estar relacionada com os processos inflamatórios (dermatites e otites) observados ao exame clínico.

Por meio da avaliação dos resultados da urinálise $(n=64)$, foi possível observar $60,94 \%$ de alteração na densidade, indicando aumento do valor comparado aos valores de referência (1.015 - 1.045) (Kaneko et al., 1997), a média e desvio padrão foram iguais a $1.043,23 \pm 11,71$. Entre as causas de aumento de densidade estão a redução de ingestão de água, desidratação, temperatura elevada, febre, hiperventilação e infecção do trato urinário (Thrall, 2007). Além disso, foram observados alterações do aspecto da urina (turvo) e presença de bactérias em $31,25 \%$ das amostras.

O exame coproparasitológico foi realizado em 55 amostras, e os resultados demonstraram que $65,45 \%$ dos animais foram diagnosticados positivos, sendo que $52 \%$ apresentaram Ancylostoma sp., assim como no trabalho realizado por Ferreira et al. (2009), e 10,9\% apresentaram Ancylostoma sp. e Trichuris sp.

A pesquisa de hemoparasitas foi realizada em 73 amostras, e $1,17 \%$ delas, apresentaram Anaplasmaplatys. Moderada infestação parasitária (pulgas ou nematóides) pode rapidamente causar uma anemia por deficiência de ferro, principalmente no cão jovem (Thompson et al., 1989).

Andrades et al. (2008), correlacionou resultados de cães apresentando endoparasitose e hemograma, e observou-se que o parasitismo não estava relacionado com a anemia, corroborando os resultados desta pesquisa, na qual não foi encontrada significância na correlação entre animais anêmicos e presença de parasitose $(p>0,05 ; n=12)$.

\section{Conclusão}

Os cães provenientes de abrigo apresentaram alterações importantes nos exames realizados, sugerindo que possuem perfis clínico e laboratorial próprios. Os valores de contagem de leucócitos, neutrófilos e bastonetes, podem ser maiores quando comparados aos valores de literatura. Não houve registros de comprometimentos durante o procedimento cirúrgico bem como no pósoperatório, provavelmente porque as alterações dos parâmetros eram causadas por deficiência nutricional, já que o baixo escore corporal pode indicar presença de anemia no cão de abrigo. Portanto, sugere-se que a avaliação pré-operatória seja composta no mínimo de exame clínico, hemograma e dosagem de albumina. 


\section{Referências}

AMARAL, A. S.; GASPAR, L. F. J.; HENNEMANN, C. R. A. Valores de Referência de Constituintes Bioquímicos Séricos para Cães da Região de Santa Maria, RS. Revista da FZVA, v. 2, n. 1, p. 86-97, 1995.

ANDRADES, A. O. et al. Incidência de Endoparasitas e sua Correlação com o Hemograma de Cães Internados no Hospital Veterinário da Universidade Federal de Santa Maria. In: CONGRESSO BRASILEIRO DE MEDICINA VETERINÁRIA, 2008. Anais do Congresso Brasileiro de Medicina Veterinária, Rio Grande do Sul: 2008.

BEITZ, D. C. et al. Your Dog's Nutritional Needs: a Science-based guide for pet owners. USA, 2006.

CERQUEIRA, C. R. E. Contributo para a promoção da saúde e do bem-estar animal em instituições de abrigo. 2012. $171 \mathrm{f}$. Dissertação (Mestrado em Medicina Veterinária) - Faculdade de Medicina Veterinária, Universidade Técnica de Lisboa, Lisboa-PT, 2012.

DRUMOND, M. R. S. Ocorrência, classificação e fatores de risco de anemia em cães. 2013. 84 f. Dissertação (Mestrado em Medicina Veterinária) - Universidade Federal de Viçosa, Minas Gerais-MG, 2013.

FERREIRA, M. A. S. et al. Avaliação de endoparasitos em cães domiciliados, de abrigo e errantes na cidade de Aracaju Sergipe. Medicina Veterinária, v. 3, n. 3, p. 20-25, 2009.

FOSSUM, T. W. Cirurgia de Pequenos Animais. 2. ed. São Paulo: Editora Roca, 2005, 1408p.
KANEKO, J. J.; HARVEY, J. W.; BRUSS, M. L. Clinical Biochemisty of Domestic Animals. 5. ed. New York: Academic Press, 1997, 932 p.

KIT LABTEST®. 2014. Instruções de uso. Minas Gerais: Labtest Diagnóstica $S A, 1 p$.

LOPES, S. T. A. et al. Valores de referência do tempo de protrombina (TP) e tempo de tromboplastina parcial ativada (TTPA) em cães. Ciência Rural, v. 35, n. 2, p. 381-384, 2005. LOPES, S. T. A.; BIONDO, A. W.; SANTOS, A. P. Manual de patologia clínica veterinária. 3. ed. Santa Maria: UFSM, 2007, 107 p. LUSTOZA, M. D. et al. Avaliação dos valores de cálcio sérico ionizado pelo método eletrodo íon seletivo em cães hígidos. Arquivo Brasileiro de Medicina Veterinária e Zootecnia, v. 57, n. 2, p. 177-180, 2005.

MILLER, L. Dog and cat care in the animal shelter. In: Miller L \& Zawistowski S (Eds.). Shelter Medicine for Veterinarians and Staff. Ames: Blackwell Publishing, 2004, p. 98-118.

NOBRE, M. C. et al. Escore de condição corporal como indicador do prognóstico de gatos com doença renal crônica. Ciência Rural, v. 40 , n. 2, p. 365-370, 2010.

THOMPSON, D. J.; SUTTON, J. B.; CHANDLER, E. A. Medicina e Terapêutica de Caninos. São Paulo: Editora Manole, 1989, 610 p.

THRALL, M. A. Hematologia e bioquímica clínica veterinária. São Paulo: Roca, 2007, 582 p.

YAMAMOTO, K. C. M. Avaliação fisiológica e comportamental de cães utilizados em terapia assistida por animais (TAA). Arquivo Brasileiro de Medicina Veterinária e Zootecnia, v. 64, n. 3, p. 568576, 2012. 